in $31 \%$, CSF protein elevation in $51 \%$, and CSF glucose was decreased in $12 \%$. MRI revealed white matter abnormalities in frontal or parietal lobes, or periventricularly, enlarged ventricles, and lesions in gray matter, brainstem, basal ganglia, and corpus callosum. EEG abnormalities included diffuse or focal slowing, and spike wave discharges. Brain biopsy of 2 patients with encephalitis showed chronic leptomeningeal inflammation and PVB19 DNA. Duration of CNS manifestations was 38 days (range, 2-198 days). Treatment with IV immunoglobulin (IG), with or without steroids, in 13 patients resulted in improvement in 8 $(61 \%)$, and none of the patients who received IG or steroids died. Neurologic sequelae occurred in $22 \%$ of 77 patients with known outcome in the total group, some improvement occurred in $16 \%$, and $56 \%$ recovered completely. Prevalence of sequelae was not different in immunocompetent patients compared to those with altered immunity, or in those with CNS manifestations, treated or untreated with IG with or without steroids. (Douvoyiannis M, Litman N, Goldman DL. Neurologic manifestations associated with parvovirus B19 infection. Clin Infect Dis June 2009;48:000). (Response and reprints: Dr Douvoyiannis. Email: mdouvoyi@montefiore.org).

COMMENT. The clinical manifestations of parvovirus B19 infection, listed in the AAP Red Book $27^{\text {th }}$ ed, 2006, include erythema infectiosum (fifth disease), polyarthropathy, chronic anemia, aplastic crisis (with sickle cell anemia), and hydrops fetalis. The current article provides an extensive review of the neurological complications of PVB19 infection.

Parvovirus B19 infection is most commonly recognized by a distinctive rash, preceded for 7 to 10 days by a mild nonspecific illness consisting of fever, myalgia, and headache. The rash is intensely red, affecting the cheeks, trunk and extremities. The incubation period is 4 to 14 days.

\title{
WEST NILE VIRUS NEUROINVASIVE DISEASE
}

Epidemiological features of West Nile Virus (WNV) disease among children $(<18$ years of age) reported to the Centers for Disease Control and Prevention from 1999 through 2007 were analyzed and compared with those of adult WNV neuroinvasive disease (WNND), in a study at CDC\&P, Fort Collins, CO. Of 1478 pediatric WNV cases reported, $443(30 \%)$ had WNND, 1009 (68\%) were classified as WN fever, and $26(2 \%)$ were unclassified. The majority had onset between July and September. Among all cases of WNND reported, only $4 \%$ were in children. Of the 443 cases of pediatric WNND, 208 (47\%) presented as meningitis, $163(37 \%)$ as encephalitis or meningoencephalitis, $5(1 \%)$ as acute flaccid paralysis (AFP), and $67(15 \%)$ as unspecified WNND. The median age of WNND cases was 12 years. Three patients died. Median annual incidence of WNND was 0.07/100,000 children, in 40 states, primarily in South Dakota, Wyoming, and New Mexico. Of a total 11,081 WNND cases in the US, 4\% occurred in children. The proportion classified as WNND was $30 \%$ and the same in children $(<18 \mathrm{yrs})$ as in young adults (18-49 yrs). Older adults $(>50$ yrs) with WNND were more often classified with encephalitis $(59 \%)$ than meningitis (23\%), whereas in children and young adults, meningitis was preponderant $(47 \%$ and 51\%, respectively). (Lindsey NP, Hayes EB, Staples JE, Fischer M. West Nile virus disease in children, United States, 1999-2007. Pediatrics June 2009;123:e1084-e1089). (Respond: Nicole P Lindsey MS, Division of Vector-Borne Infectious Diseases, Centers for 
Disease Control and Prevention, 3150 Rampart Road, Fort Collins, CO 80522. E-mail: frd3@cdc.gov).

COMMENT. According to the AAP Red Book (2006), the majority of pediatric WNV infections are asymptomatic, $20 \%$ have WNF, and $<1 \%$ develop neuroinvasive disease. Risk of WNND increases with age and is highest in adults $>60$ years. Patients with WNND present with neck stiffness and headache, typical of aseptic meningitis, mental status changes of encephalitis, movement disorders such as tremor, seizures, or acute flaccid paralysis clinically similar to poliomyelitis or Guillain-Barre syndrome.

\section{SEIZURE DISORDERS}

\section{TWINKLE MUTATIONS AND REFRACTORY STATUS EPILEPTICUS}

Severe epileptic encephalopathy and refractory status epilepticus are reported in a 20year follow-up of 23 patients with recessive Twinkle mutations studied at Helsinki University Central Hospital, Finland. Dominant mutations of the C10orf2 gene are linked with progressive external ophthalmoplegia, while recessive mutations cause mitochondrial DNA depletion and encephalopathy or hepatoencephalopathy, similar to phenotypes associated with recessive POLG1 mutations (eg Alpers syndrome). The authors had previously described infantile onset spinocerebellar ataxia caused by Twinkle mutations. On long-term follow-up, further clinical phenotypes developed, including refractory status epilepticus, migraine-like headaches, and psychoses. Myoclonic or focal clonic seizures occurred in 18 patients, progressed to epilepsia partialis continua in 15 and generalized status epilepticus in 13, 8 of whom died. The first episode of status epilepticus occurred between 15 and 34 years of age in homozygotes, and at 2 and 4 years of age in the compound heterozygotes. Status lasted from several days to weeks. Valproate caused elevation of liver enzymes in 2 patients and was discontinued. Phenytoin or fosphenytoin was ineffective and caused an elevation of liver enzymes. Oxcarbazepine, lamotrigine or levetiracetam was of benefit in some. MRI showed focal, stroke-like lesions, some hemispheric, resulting in edema, necrosis, and brain atrophy, including the hippocampus, and confirmed on neuropathology exam. (Lonnqvist T, Paetau A, Valanne L, Pihko H. Recessive twinkle mutations cause severe epileptic encephalopathy. Brain June 2009;132:1553-1562). (Respond: Dr Tuula Lonnqvist, Division of Child Neurology, Helsinki University Central Hospital, PO Box 280, Helsinki, 00029 Finland. E-mail: tuula.lonnqvist@,hus.fi).

COMMENT. The authors comment that the infantile onset spinocerebellar ataxia (IOSCA) syndrome shares features with other mitochondrial recessive ataxia syndromes, including Friedreich's ataxia, mitochondrial spinocerebellar ataxia-epilepsy (MSCAE) syndrome and mitochondrial DNA polymerase gamma (POLG)-Alpers syndrome. IOSCA, a progressive neurodegenerative disease, is caused by homozygous or compound heterozygous C10orf2 gene mutations that code for the mitochondrial DNA helicase Twinkle. Status epilepticus, migraine-like headaches and psychiatric symptoms are also pathognomonic for the disease. These symptoms should alert the clinician to consider a mitochondrial encephalopathy in differential diagnosis. 